\title{
A coupled model for government communication and rumor spreading in emergencies
}

\author{
Jiuping $\mathrm{Xu}^{1 *}$, Mengxiang Zhang ${ }^{1}$ and Jingneng $\mathrm{Ni}^{1,2}$
}

\section{"Correspondence:}

xujiuping@scu.edu.cn

'Uncertainty Decision-Making

Laboratory, Sichuan University,

Yihuan Road, Chengdu, 610064,

China

Full list of author information is

available at the end of the article

\begin{abstract}
The dissemination of official messages and the spreading of rumors are both key factors in emergency situations. In this paper, a coupled model is established to describe the interaction between official government communiques and the spreading of rumors during emergencies. The mathematical property of the proposed model is discussed, and the results showed that the official message diffusion has positive effects on thwarting rumor spreading. Three different scenarios for population behavior evolution are identified according to the degree of government involvement. The results shed new light to comprehend information dissemination in emergencies and provide some hint on how to limit the spreading of rumor within populations after emergency occur.
\end{abstract}

Keywords: coupled model; government communication; rumor spreading; emergency event

\section{Introduction}

Information dissemination is one of the key factors in the evolution of emergency incidents. After the occurrence of an emergency event, the affected and nonaffected public is eager for information [1], and, as a result, rumors tend to appear through informal and unofficial channels [2]. On the one hand, these rumors have been shown to have a negative influence on people's lives, leading to possible financial loss, feelings of panic, and irrational behavior [3]. Such negativity from unsubstantiated rumors can develop into extreme public reactions and cause panic during emergencies [4]. On the other hand, such rumors have been shown to have possible positive effects on the evolution of public opinion since they can encourage authorities to actively respond to the crisis [5] to maintain social stability [6]. Official dissemination of emergency event information through official and sanctioned channels can have a positive effect on the public's behavior since false rumors can be quashed and the true information confirmed. The rapid dissemination of government information, therefore, is an effective and essential measure to combat the spread of false rumors and to guide public opinion. From this analysis it can be seen that in emergency management there exists a coupling mechanism between official government information dissemination and the spreading of rumors, both of which impact the

(c) $2016 \mathrm{Xu}$ et al. This article is distributed under the terms of the Creative Commons Attribution 4.0 International License (http://creativecommons.org/licenses/by/4.0/), which permits unrestricted use, distribution, and reproduction in any medium, provided you give appropriate credit to the original author(s) and the source, provide a link to the Creative Commons license, and indicate if changes were made. 
evolution of group crisis behavior. Since communication management is the core of crisis response [7], there is a need to examine these coupling effects during anomalous events.

In various studies on emergency information dissemination, the topic of epidemic spreading has inspired a great deal of rumor spreading for many years [8], especially the SIR model. Daley and Kendall [9] first introduced a classical rumor spreading model in the 1960s, and since then this quantitative model has been substantially extended [1012]. Maki and Thomson [13] analyzed a rumor-spreading mechanism and developed the MK model. A number of studies have proposed more complex rumor-spreading models in large social networks [14-16]. However, quantitative studies of government information dissemination have been fewer. Huo et al. [17] used a logistic model to describe an official information dissemination process but neglected to examine the effect of rumors.

Little previous research, however, has examined the interaction between government communication mechanisms and the spreading of rumors under extreme conditions. Zhang et al. [5] used a trimolecular model to explore the relationship between rumor spreading and emergency development. Huo et al. [17] applied Wang's system to model an interplay effect between official action and rumor spreading. Zhao et al. [18] analyzed an interplay mechanism amongst the official media, rumor dissemination, and emergency evolution based on the ideas of the trimolecular model. These research studies used abstract functions, such as social utility, to measure the impact of the authorities' action and the spread of rumors, but ignored the effect of the dissemination of official messages and rumors after emergency incidents on the people. Because in such emergencies individual behavior can affect group behavior, it is necessary to explore how official message dissemination and rumor spreading impact the evolution of population behavior.

In this paper, the impact on emergency group behavior of the interplay between official message dissemination and rumor spreading over multiple channels is examined. This study develops a government communication and rumor spreading coupled model after an emergency incident from the perspective of the affected population. The objectives of this study are: (1) to extend classical models of spreading epidemics to describe the coupling mechanism for government communication and rumor spreading in an emergency; (2) to use mathematical analyses to discuss a series of key factors in interaction between the two kinds of information diffusion; and (3) to investigate the evolution of population behavior under different degrees of government involvement.

The remainder of this paper is organized as follows. The coupled model for government communication and rumor spreading is established in Section 2. In Section 3, the mathematical property of the proposed system is analyzed based on the local and global theory, and three different scenarios for population evolution are interpreted according to the different degrees of government involvement. Some results are discussed in Section 4. Conclusions and recommendations for further research are given in the final section.

\section{Modeling}

In this section, a coupled model for communication and rumor spreading under an emergency situation is constructed. Firstly, the coupled mechanism is introduced. Then, a conceptual model is given before the mathematical model is constructed based on the foundation. 


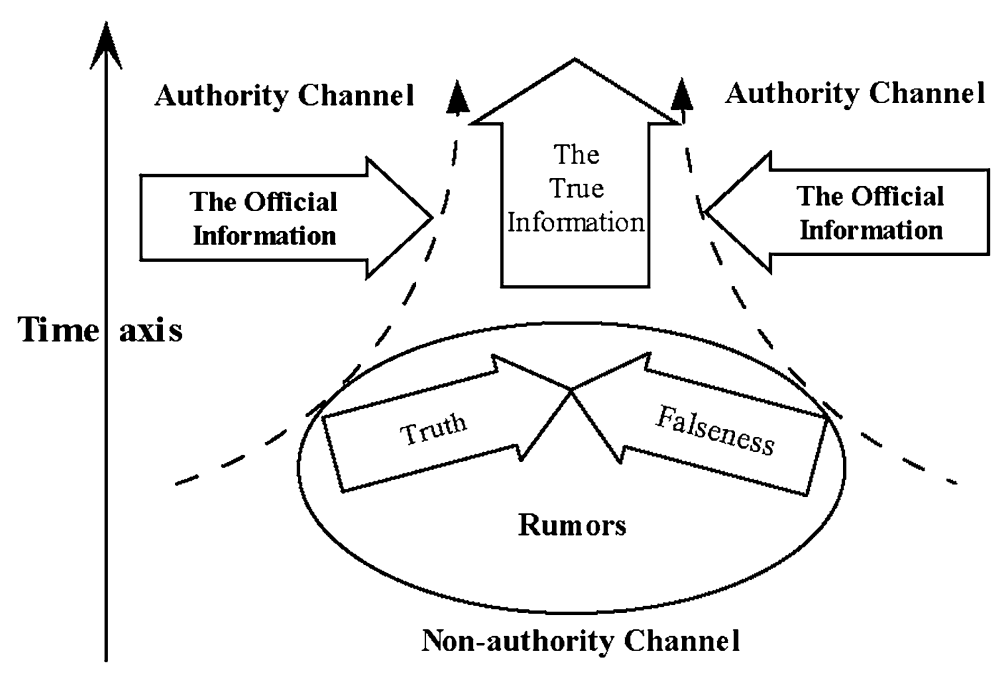

Figure 1 Government communication and rumor spreading coupling mechanism.

\subsection{Government communication and rumor-spreading coupled mechanism}

There are two information dissemination effects in the different channels, and each of the two channels has its own main dissemination styles. Government communication uses official channels through which official messages are issued to effectively dispel public suspicion and to guide public opinion toward the maintenance of social stability. Rumor spreading, on the other hand, uses nonofficial channels through which individuals fabricate and transmit rumors about the emergency to fill the gap between their anxiety and the lack of substantive information from official channels. Rumors are often mixtures of true and false messages, and there exists a competition between these two sides as the rumor is spread since people with a critical ability will separate the truth from the speculation when they spread the rumor, whilst those who are unable to use critical ability to test the truth of the rumors will pass on the rumor without judgment, often exacerbating the falseness. This competitive rumor transmission across the unofficial channels can shape public opinion and cause significant social unrest.

However, a coupling mechanism exists between the rumor spreading and the government communication since the issuing of official messages to explain the emergency situation may break the competitive rumor spreading by compelling official channels to make open responses as quickly as possible. These interactive processes between government communication and rumor spreading are shown in Figure 1.

\subsection{Conceptual model}

Rumor spreading could be seen to be similar to the spread of infectious diseases in populations [19]. A closed and homogeneous population consisting of $M$ individuals is considered under an emergency situation. As shown in Figure 2, the population is divided into three agents: the unaware $(U)$, the supporter $(S)$, and the nonsupporter $(N)$. Here $U$ stands for those agents who have not heard any information (either official of unofficial) about the emergency event and are information demanders. $S$ represents the agents who have the ability to judge the truth of the rumors they hear, or who believe the official voices they hear, and who spread true messages about the public incidents to others. Their behavior positively influences the spread of the truth of the emergency and strongly supports 


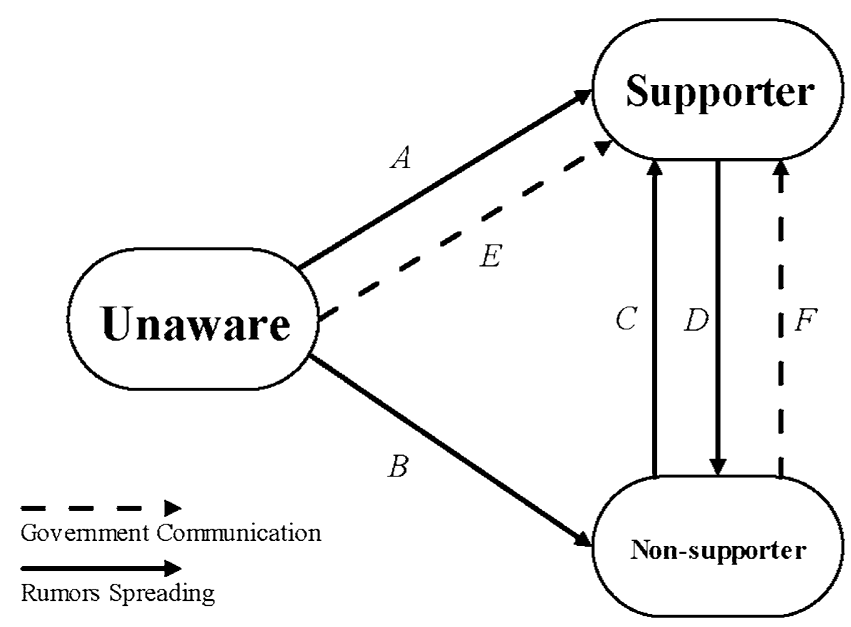

Figure 2 Flow diagram for information dissemination in official and unofficial channels.

Table 1 The process of conversion relation under information diffusion

\begin{tabular}{lll}
\hline Process of conversion & Reason of conversion & Explanation for conversion \\
\hline$A$ & The truth of the rumors & The unaware become supporters \\
$B$ & The falseness of the rumors & The unaware become nonsupporters \\
$C$ & The truth of the rumors & The nonsupporters become supporters \\
$D$ & The falseness of the rumors & The supporters become nonsupporters \\
$E$ & The government communication & The unaware become supporters \\
$F$ & The government communication & The nonsupporters become supporters \\
\hline
\end{tabular}

government action in coping with the emergency. $N$ denotes those agents of the public who are unable to judge the truth of rumors they hear or who disbelieve the authority's messages, thus often passing on false information to others. Their behavior does not support the government's response to the emergency. Under this assumption, $U(t), S(t)$, and $N(t)$ denote the density of population that are unaware, supporters, and nonsupporters at time $t$, respectively.

Based on such a foundation, the emergency information received by the unaware, the supporters, and the nonsupporters is a combination from official and nonofficial channels. The extent of government communication and rumor spreading can influence the conversion of the three kinds of agents. The concrete conversion relationships between the three kinds of members are shown in Figure 2 and Table 1. The arrowheads denote the direction of the members. The arrowheads $E$ and $F$ represent the effect of the official message dissemination, the arrowheads $A, B, C$, and $D$ denote the effect of the rumor spreading, $C$ and $D$ represent the competitive rumor spreading process, and all arrowheads represent the coupling effect of the government communication and rumor transmission channels.

\subsection{Mathematical model}

The information dissemination process can be represented by a system of differential equations [20], which is modeled to describe the coupling process of government communication and rumor spreading in an emergency situation. As shown in Figure 2, this model consists of two components, the government communication process and the rumor spreading process. For government communication, the official message can reach 
Table 2 Basic parameters

\begin{tabular}{ll}
\hline Parameter & Meaning \\
\hline$\alpha_{1}$ & The true rumor dissemination rate \\
$\alpha_{2}$ & The false rumor dissemination rate \\
$\gamma$ & The rumor spreading competition impact coefficient \\
$\beta_{1}$ & The official message dissemination rate for the unaware \\
$\beta_{2}$ & The official message dissemination rate for the nonsupporter \\
$m$ & The threshold of the rumor spreading competition \\
\hline
\end{tabular}

everyone (including the unaware, supporters, and nonsupporters) through the authorities' media channels. Rumors are disseminated through the direct contact of the spreaders (including supporters and nonsupporters) with others. The parameter list for the model is described in Table 2. These parameters are constants, and the values for $\alpha_{1}, \alpha_{2}, \beta_{1}, \beta_{2}$, $\gamma$, and $m$ are in the range $[0,1)$.

According to the definition for $U(t), S(t)$, and $N(t)$, it is obvious that they satisfy the normalization condition

$$
U(t)+S(t)+N(t)=1 \quad \text { and } \quad U(t), S(t), N(t) \geq 0 .
$$

\section{The government communication process}

Government communications usually have high credibility and a wide supporter base. Based on the analysis of the conceptual model, the conversion processes $E$ and $F$ represent the extent to which the influence of the official message dissemination on an unaware or a nonsupporter is likely to move them to become a supporter, as seen in Figure 2. According to hypotheses 1,2 , and 3 , we use $\beta_{1}$ to express the probability that an unaware who has heard the official information becomes a supporter. Therefore, $\beta_{1} U(t)$ is the rate of $S(t)$ growth that the unaware requires from the influence of the official information dissemination to become a supporter. Similarly, $\beta_{2}$ and $\beta_{2} N(t)$ represent the probability, and $S(t)$ the growth rate required for a nonsupporter to become a supporter through official information dissemination. In general, it can be observed that $\beta_{1}$ is greater than $\beta_{2}$.

Consequently, the government communication process is governed by the system

$$
\left\{\begin{array}{l}
\frac{d U(t)}{d t}=-\beta_{1} U(t) \\
\frac{d S(t)}{d t}=\beta_{1} U(t)+\beta_{2} N(t), \\
\frac{d N(t)}{d t}=-\beta_{2} N(t)
\end{array}\right.
$$

\section{The rumor-spreading process}

Many rumors are spread through nonofficial channels, and there is competition between these channels. As shown in Figure 2, the arrowheads $A, B, C$, and $D$ denote the conversion process for rumor circulation within which the competitive rumor spreading mechanism is denoted by arrowheads $C$ and $D$. According to hypotheses $1,2,4$, and 5 , the rumorspreading model is as follows.

(1) When an unaware comes into contact with a supporter, the unaware becomes a supporter with probability $\alpha_{1}$, namely the true rumor spreading rate; $\alpha_{1} U(t) S(t)$ is the rate of $S(t)$ growth that the unaware needs to become a supporter through the information spread via nonofficial channels. 
(2) When an unaware contacts a nonsupporter, the unaware becomes a nonsupporter with probability $\alpha_{2}$, namely the false rumor spreading rate; $\alpha_{2} U(t) N(t)$ is the rate of $N(t)$ growth that the unaware needs to become a nonsupporter through the information spread via nonofficial channels.

(3) There is competition between the true and false rumor-spreading rates [21]. To depict this competitive mechanism, based on the interactive characteristics of the rumors spread through nonofficial channels [22, 23], we consider a threshold rate $m$ for the competitive information process. Then $\gamma[m-S(t)] S(t) N(t)$ denotes the rate of competition between the true and false rumors, and the impact coefficient is $\gamma$. The equality $\gamma[m-S(t)] S(t) N(t)>0$, that is $S(t)<m$, indicates that the scale of influence of the false rumor spreading is greater than that of the truth dissemination, and the number of supporters who will become nonsupporters is greater than that of the nonsupporters who are willing to become a supporter; inversely, $S(t)>m$ indicates that the true rumor spread has a greater influence than the false dissemination.

Consequently, the rumor spreading process is governed by the system

$$
\left\{\begin{array}{l}
\frac{d U(t)}{d t}=-\alpha_{1} U(t) S(t)-\alpha_{2} U(t) N(t) \\
\frac{d S(t)}{d t}=\alpha_{1} U(t) S(t)-\gamma[m-S(t)] S(t) N(t) \\
\frac{d N(t)}{d t}=\alpha_{2} U(t) N(t)+\gamma[m-S(t)] S(t) N(t)
\end{array}\right.
$$

\section{The coupled model for government communication and rumor spreading}

For the government communication and rumor-spreading coupling mechanism, there are two information dissemination processes affecting the communication. As shown in Figure 2, all arrowheads denote this interactive process. Arrowheads $E$ and $F$ represent the voice of authority, and it can be seen that these break the balance of the arrowheads $A, B, C$, and $D$. According to hypotheses $1,2,3,4$, and 5 and based on the above analysis of the official messages and rumor dissemination processes, the increase equation for the member $U$ is

$$
\frac{d U(t)}{d t}=-\alpha_{1} U(t) S(t)-\alpha_{2} U(t) N(t)-\beta_{1} U(t),
$$

and the increase equation for the member $S$ is

$$
\frac{d S(t)}{d t}=\alpha_{1} U(t) S(t)-\gamma[m-S(t)] S(t) N(t)+\beta_{1} U(t)+\beta_{2} N(t)
$$

Similarly, the increase equation for the member $N$ is

$$
\frac{d N(t)}{d t}=\alpha_{2} U(t) N(t)+\gamma[m-S(t)] S(t) N(t)-\beta_{2} N(t) .
$$

So, the model proposed from the preceding analysis is described by the system

$$
\left\{\begin{array}{l}
\frac{d U(t)}{d t}=-\alpha_{1} U(t) S(t)-\alpha_{2} U(t) N(t)-\beta_{1} U(t) \\
\frac{d S(t)}{d t}=\alpha_{1} U(t) S(t)-\gamma[m-S(t)] S(t) N(t)+\beta_{1} U(t)+\beta_{2} N(t) \\
\frac{d N(t)}{d t}=\alpha_{2} U(t) N(t)+\gamma[m-S(t)] S(t) N(t)-\beta_{2} N(t)
\end{array}\right.
$$

where $0 \leq U(t), S(t), N(t) \leq 1, U(t)+S(t)+N(t)=1$, and $0 \leq \alpha_{1}, \alpha_{2}, \beta_{1}, \beta_{2}, \gamma, m<1$. 
After the emergency occurs, the agents of individuals with supportive or nonsupportive behavior toward the government is a key to the measure of the deterioration of events, so this proportion is one of significant aspects of emergency situations that governments need to pay attention to. Therefore, a planar system for the supporter and nonsupporter is now discussed. Because the unaware $U$, the supporter $S$, and the nonsupporter $N$ satisfy the normalization condition $U(t)+S(t)+N(t)=1$, the planar system for the coupling mechanism is described as follows:

$$
\left\{\begin{aligned}
\frac{d S(t)}{d t}= & \gamma S(t)^{2} N(t)-\left(\gamma m+\alpha_{1}\right) S(t) N(t)-\alpha_{1} S(t)^{2} \\
& +\left(\alpha_{1}-\beta_{1}\right) S(t)+\left(\beta_{2}-\beta_{1}\right) N(t)+\beta_{1} \\
\frac{d N(t)}{d t}= & -\gamma S(t)^{2} N(t)+\left(\gamma m-\alpha_{2}\right) S(t) N(t)-\alpha_{2} N(t)^{2}+\left(\alpha_{2}-\beta_{2}\right) N(t)
\end{aligned}\right.
$$

where $0 \leq S(t), N(t) \leq 1, S(t)+N(t) \leq 1$, and $0 \leq \alpha_{1}, \alpha_{2}, \beta_{1}, \beta_{2}, \gamma, m<1$

\section{Results and analysis}

In this section, a mathematical analysis for the coupled model is introduced. Then, based on the results of this analysis, three different scenarios for our model are proposed to interpret the coupled effect of government communication and rumor spreading in emergencies according to different degrees of government involvement.

\subsection{Mathematical analysis}

Based on the local and global theory of differential systems, the property of the coupling system is discussed, which reflects the final results for the government communication and rumor-spreading interaction. We prove several theorems and remarks.

Theorem 1 When $\alpha_{1}+\alpha_{2} \geq \gamma$, there is no closed orbit in system (5).

Proof Let

$$
\left\{\begin{array}{l}
F_{1}=\gamma S(t)^{2} N(t)-\left(\gamma m+\alpha_{1}\right) S(t) N(t)-\alpha_{1} S(t)^{2}+\left(\alpha_{1}-\beta_{1}\right) S(t)+\left(\beta_{2}-\beta_{1}\right) N(t)+\beta_{1} \\
G_{1}=-\gamma S(t)^{2} N(t)+\left(\gamma m-\alpha_{2}\right) S(t) N(t)-\alpha_{2} N(t)^{2}+\left(\alpha_{2}-\beta_{2}\right) N(t)
\end{array}\right.
$$

and definite the Dulac function $B_{1}=\frac{1}{S(t) N(t)}$. Then

$$
\begin{aligned}
D_{1} & =\frac{\partial}{\partial S(t)}\left(B_{1} F_{1}\right)+\frac{\partial}{\partial N(t)}\left(B_{1} G_{1}\right) \\
& =\gamma-\alpha_{1} \frac{1}{N(t)}-\alpha_{2} \frac{1}{S(t)}-\left[\beta_{1}\left(\frac{1}{N(t)}-1\right)+\beta_{2}\right] \frac{1}{S(t)^{2}} .
\end{aligned}
$$

Because $0 \leq S(t), N(t) \leq 1$, we have $\frac{1}{S(t)}, \frac{1}{N(t)} \geq 1$, so that

$$
\alpha_{1} \frac{1}{N(t)}+\alpha_{2} \frac{1}{S(t)}>\alpha_{1}+\alpha_{2} \geq \gamma \quad \text { and } \quad\left[\beta_{1}\left(\frac{1}{N(t)}-1\right)+\beta_{2}\right] \frac{1}{S(t)^{2}}>0
$$

Therefore, $D_{1}<0$. It follows from the Bendixson-Dulac principle [24] that there is no closed orbit in system (5) if $\alpha_{1}+\alpha_{2} \geq \gamma$.

Theorem 2 System (5) has a node A(1,0), which is asymptotically stable. 
Proof The steady state of system (5) satisfies the following system of equations:

$$
\left\{\begin{array}{l}
\gamma S(t)^{2} N(t)-\left(\gamma m+\alpha_{1}\right) S(t) N(t)-\alpha_{1} S(t)^{2}+\left(\alpha_{1}-\beta_{1}\right) S(t)+\left(\beta_{2}-\beta_{1}\right) N(t)+\beta_{1}=0, \\
-\gamma S(t)^{2} N(t)+\left(\gamma m-\alpha_{2}\right) S(t) N(t)-\alpha_{2} N(t)^{2}+\left(\alpha_{2}-\beta_{2}\right) N(t)=0 .
\end{array}\right.
$$

It is easy to visualize that system (5) always has a fixed point $(1,0)$. Then, the coefficient matrix for the linear system of system (5) is

$$
B=\left[\begin{array}{cc}
\left(\alpha_{1}-\beta_{1}\right)-\left(\gamma m+\alpha_{1}\right) N(t)-2 \alpha_{1} S(t)+2 \gamma S(t) N(t) & \left(\beta_{2}-\beta_{1}\right)-\left(\gamma m+\alpha_{1}\right) S(t)+\gamma S(t)^{2} \\
-\left(\gamma m-\alpha_{2}\right) N(t)-2 \gamma S(t) N(t) & \left(\alpha_{2}-\beta_{2}\right)+\left(\gamma m-\alpha_{2}\right) S(t)-2 \alpha_{2} N(t)-\gamma S(t)^{2}
\end{array}\right] .
$$

After calculation, the coefficient matrix for $A(1,0)$ is

$$
B_{A}=\left[\begin{array}{cc}
-\alpha_{1}-\beta_{1} & \beta_{2}-\beta_{1}+\gamma(1-m)-\alpha_{1} \\
0 & \gamma(m-1)-\beta_{2}
\end{array}\right]
$$

with

$$
\begin{aligned}
& \operatorname{Tr} B_{A}=-\alpha_{1}-\beta_{1}+\gamma(m-1)-\beta_{2}, \\
& \operatorname{Det} B_{A}=\left(\alpha_{1}+\beta_{1}\right)\left[\gamma(1-m)+\beta_{2}\right],
\end{aligned}
$$

and

$$
\begin{aligned}
\Delta & =\left[\alpha_{1}+\beta_{1}+\gamma(1-m)+\beta_{2}\right]^{2}-4\left(\alpha_{1}+\beta_{1}\right)\left[\gamma(1-m)+\beta_{2}\right] \\
& =\left[\alpha_{1}+\beta_{1}-\gamma(1-m)-\beta_{2}\right]^{2} \geq 0 .
\end{aligned}
$$

Because $0 \leq \alpha_{1}, \alpha_{2}, \beta_{1}, \beta_{2}, \gamma, m<1$, we have $\operatorname{Tr} B_{A}<0$ and $\operatorname{Det} B_{A}>0$. So, the fixed point $A(1,0)$ is an asymptotically stable node in terms of the local theory of differential system [24].

Theorem 3 When $k=(\gamma m)^{2}-4 \gamma \beta_{2}>0$, system (5) has a node $B\left(M_{1}, 1-M_{1}\right)$ and a saddle point $C\left(M_{2}, 1-M_{2}\right)$, where $M_{1}=\frac{m}{2}-\frac{\sqrt{k}}{2 \gamma}, M_{2}=\frac{m}{2}+\frac{\sqrt{k}}{2 \gamma}$; the node $B\left(M_{1}, 1-M_{1}\right)$ is asymptotically stable.

Proof The steady state of system (5) satisfies the following system of equations:

$$
\left\{\begin{array}{l}
\gamma S(t)^{2} N(t)-\left(\gamma m+\alpha_{1}\right) S(t) N(t)-\alpha_{1} S(t)^{2}+\left(\alpha_{1}-\beta_{1}\right) S(t)+\left(\beta_{2}-\beta_{1}\right) N(t)+\beta_{1}=0 \\
-\gamma S(t)^{2} N(t)+\left(\gamma m-\alpha_{2}\right) S(t) N(t)-\alpha_{2} N(t)^{2}+\left(\alpha_{2}-\beta_{2}\right) N(t)=0 .
\end{array}\right.
$$

Assuming that $S(t)+N(t)=1$, this equation system can be rewritten as

$$
\left\{\begin{array}{l}
N(t)\left[\gamma S(t)^{2}-\gamma m S(t)+\beta_{2}\right]=0 \\
-N(t)\left[\gamma S(t)^{2}-\gamma m S(t)+\beta_{2}\right]=0
\end{array}\right.
$$

Therefore, $B\left(M_{1}, 1-M_{1}\right)$ and $C\left(M_{2}, 1-M_{2}\right)$ are the fixed points of system (5), where $M_{1}$, $M_{2}$ satisfy the following conditions respectively:

$$
\gamma M_{1}^{2}-\gamma m M_{1}+\beta_{2}=0, \quad 0 \leq M_{1} \leq 1,
$$




$$
\gamma M_{2}^{2}-\gamma m M_{2}+\beta_{2}=0, \quad 0 \leq M_{2} \leq 1 .
$$

Solving these quadratic equations, we get

$$
M_{1}=\frac{m}{2}-\frac{\sqrt{(\gamma m)^{2}-4 \gamma \beta_{2}}}{2 \gamma}, \quad M_{2}=\frac{m}{2}+\frac{\sqrt{(\gamma m)^{2}-4 \gamma \beta_{2}}}{2 \gamma},
$$

where $(\gamma m)^{2}-4 \gamma \beta_{2} \geq 0$. Under the assumption $k=(\gamma m)^{2}-4 \gamma \beta_{2}>0,0 \leq \beta_{2}<1$, we have $0<\sqrt{k}<\frac{m}{2}$.

Therefore,

$$
0<M_{1}<\frac{m}{2}, \quad \frac{m}{2}<M_{2}<m .
$$

Because system (5) is transited by system (4) and condition (1), the other two-dimensional system

$$
\left\{\begin{aligned}
\frac{d U(t)}{d t}= & \alpha_{1} U(t)^{2}+\left(\alpha_{1}-\alpha_{2}\right) U(t) N(t)-\left(\alpha_{1}+\beta_{1}\right) U(t) \\
\frac{d N(t)}{d t}= & -\gamma N(t)^{3}-2 \gamma U(t) N(t)^{2}-\gamma N(t) U(t)^{2}+\gamma(2-m) N^{2} \\
& +\left[\gamma(2-m)+\alpha_{2}\right] N(t) U(t)+\left[\gamma(m-1)-\beta_{2}\right] N(t)
\end{aligned}\right.
$$

has been transited by system (4) and condition (1), where $0 \leq U(t), N(t) \leq 1, U(t)+N(t) \leq$ 1 , and $0 \leq \alpha_{1}, \alpha_{2}, \gamma, m<1$.

Since system (6) is converted by system (4) and condition (1), the fixed point of system (5) corresponds to that of system (6). So, $B\left(M_{1}, 1-M_{1}\right)$ and $C\left(M_{2}, 1-M_{2}\right)$, which are the fixed points of system (6), correspond to the fixed points $B^{\prime}\left(0,1-M_{1}\right)$ and $C^{\prime}\left(0,1-M_{2}\right)$ of system (6).

After calculation, the coefficient matrix for the linear system of system (6) is

$$
B=\left[\begin{array}{cc}
\left(\alpha_{1}-\alpha_{2}\right) N(t)-\left(\alpha_{1}+\beta_{1}\right) & 0 \\
2 \gamma N(t)^{2}+\left[\gamma(2-m)+\alpha_{2}\right] N(t) & -3 \gamma N(t)^{2}+2 \gamma(2-m) N(t)+\gamma(m-1)-\beta_{2}
\end{array}\right] .
$$

There are the following two situations for the fixed points of system (6):

I. For $B^{\prime}\left(0,1-M_{1}\right)$, the coefficient matrix of the linear system of system (6) is

$$
B_{B}^{\prime}=\left[\begin{array}{cc}
\left(\alpha_{1}-\alpha_{2}\right)\left(1-M_{1}\right)-\left(\alpha_{1}+\beta_{1}\right) & 0 \\
2 \gamma\left(1-M_{1}\right)^{2}+\left[\gamma(2-m)+\alpha_{2}\right]\left(1-M_{1}\right) & -3 \gamma\left(1-M_{1}\right)^{2}+2 \gamma(2-m)\left(1-M_{1}\right)+\gamma(m-1)-\beta_{2}
\end{array}\right] .
$$

Because $\gamma M_{1}^{2}-\gamma m M_{1}+\beta_{2}=0$, we have

$$
\begin{aligned}
-3 \gamma & \left(1-M_{1}\right)^{2}+2 \gamma(2-m)\left(1-M_{1}\right)+\gamma(m-1)-\beta_{2} \\
= & {\left[-2 \gamma\left(1-M_{1}\right)^{2}+\gamma(2-m)\left(1-M_{1}\right)\right] } \\
& +\left[-\gamma\left(1-M_{1}\right)^{2}+\gamma(2-m)\left(1-M_{1}\right)+\gamma(m-1)-\beta_{2}\right] \\
= & \gamma\left(1-M_{1}\right)\left(2 M_{1}-m\right)+\gamma M_{1}^{2}-\gamma m M_{1}+\beta_{2} \\
= & \gamma\left(1-M_{1}\right)\left(2 M_{1}-m\right) .
\end{aligned}
$$


Therefore, the coefficient matrix of system (6) is

$$
B_{B^{\prime}}=\left[\begin{array}{cc}
\left(\alpha_{1}-\alpha_{2}\right)\left(1-M_{1}\right)-\left(\alpha_{1}+\beta_{1}\right) & 0 \\
2 \gamma\left(1-M_{1}\right)^{2}+\left[\gamma(2-m)+\alpha_{2}\right]\left(1-M_{1}\right) & \gamma\left(1-M_{1}\right)\left(2 M_{1}-m\right)
\end{array}\right]
$$

with

$$
\begin{aligned}
& \operatorname{Tr} B_{B^{\prime}}=\left(\alpha_{1}-\alpha_{2}\right)\left(1-M_{1}\right)-\left(\alpha_{1}+\beta_{1}\right)+\gamma\left(1-M_{1}\right)\left(2 M_{1}-m\right), \\
& \operatorname{Det} B_{B^{\prime}}=\left[\left(\alpha_{1}-\alpha_{2}\right)\left(1-M_{1}\right)-\left(\alpha_{1}+\beta_{1}\right)\right]\left[\gamma\left(1-M_{1}\right)\left(2 M_{1}-m\right)\right],
\end{aligned}
$$

and

$$
\Delta=\left[\left(\alpha_{1}-\alpha_{2}\right)\left(1-M_{1}\right)-\left(\alpha_{1}+\beta_{1}\right)-\gamma\left(1-M_{1}\right)\left(2 M_{1}-m\right)\right]^{2} \geq 0
$$

Because $0<M_{1}<\frac{m}{2}$ and $\alpha_{1}, \alpha_{2}, \beta_{1} \in[0,1)$, we have $2 M_{1}-m<0$ and $1-M_{1}>0$, so that

$$
\gamma\left(1-M_{1}\right)\left(2 M_{1}-m\right)<0
$$

and

$$
\begin{aligned}
& \left(\alpha_{1}-\alpha_{2}\right)\left(1-M_{1}\right)-\left(\alpha_{1}+\beta_{1}\right) \\
& \quad=-\left(\alpha_{1}+\alpha_{2}\right) M_{1}-\left(\alpha_{1}+\beta_{1}\right)<0 .
\end{aligned}
$$

Therefore, $\operatorname{Tr} B_{B^{\prime}}<0$ and Det $B_{B^{\prime}}>0$. According to local theory [24], the fixed point $B^{\prime}\left(0,1-M_{1}\right)$ is asymptotically stable, and the corresponding fixed point $B\left(M_{1}, 1-M_{1}\right)$ of system (5) is asymptotically stable.

II. Similarly, $C^{\prime}\left(0,1-M_{2}\right)$, where $\gamma M_{2}^{2}-\gamma m M_{2}+\beta_{2}=0$ and $\frac{m}{2}<M_{2}<m$, has the coefficient matrix for the linear system of system (6)

$$
B_{C^{\prime}}=\left[\begin{array}{cc}
\left(\alpha_{1}-\alpha_{2}\right)\left(1-M_{2}\right)-\left(\alpha_{1}+\beta_{1}\right) & 0 \\
2 \gamma\left(1-M_{2}\right)^{2}+\left[\gamma(2-m)+\alpha_{2}\right]\left(1-M_{2}\right) & \gamma\left(1-M_{2}\right)\left(2 M_{2}-m\right)
\end{array}\right]
$$

with

$$
\operatorname{Det} B_{C^{\prime}}=\left[\left(\alpha_{1}-\alpha_{2}\right)\left(1-M_{1}\right)-\left(\alpha_{1}+\beta_{1}\right)\right]\left[\gamma\left(1-M_{1}\right)\left(2 M_{1}-m\right)\right] \text {. }
$$

Because $\frac{m}{2}<M_{2}<m$ and $\alpha_{1}, \alpha_{2}, \beta_{1} \in[0,1)$, we have $2 M_{2}-m>0,1-M_{1}>0$,

$$
\gamma\left(1-M_{1}\right)\left(2 M_{1}-m\right)>0
$$

and

$$
\begin{aligned}
& \left(\alpha_{1}-\alpha_{2}\right)\left(1-M_{1}\right)-\left(\alpha_{1}+\beta_{1}\right) \\
& \quad=-\left(\alpha_{1}+\alpha_{2}\right) M_{1}-\left(\alpha_{1}+\beta_{1}\right)<0 .
\end{aligned}
$$


Therefore, Det $B_{C^{\prime}}<0$. According to local theory [24], the fixed point $C^{\prime}\left(0,1-M_{2}\right)$ is a saddle point, and the corresponding fixed point $C\left(M_{2}, 1-M_{2}\right)$ of system (5) is a saddle point.

According to Theorems 2, 3, we have the following remark for system (5).

Remark 1 When $\beta_{2}=0$, system (5) has three nodes $A(1,0), B(0,1), O(0,0)$ and a saddle point $C(m, 1-m)$; the nodes $A(1,0)$ and $B(0,1)$ are asymptotically stable, and the node $O(0,0)$ is unstable.

\subsection{Scenario analysis}

According to the results of the preceding local and global analysis for system (5), it follows that the parameters $\beta_{2}, \gamma, m$ determine how the system evolves in time. There exist three scenarios in terms of different parameter settings, which include $\beta_{2}=0,0<\beta_{2}<\frac{\gamma m^{2}}{4}$, and $\beta_{2} \geq \frac{\gamma m^{2}}{4}$, and all of the global phase portraits for the coupled models under different scenarios are drawn as in Figure 3 to describe the government communication and rumor-spreading coupled mechanism evolutionary process. Because the parameter $\beta_{2}$ is the official message dissemination rate for the nonsupporter and its value represents an influence degree of authoritative information thwarting the rumor spreading, there are three scenarios after public emergencies that are defined and discussed based on different values of the parameter $\beta_{2}$ : inefficacy of government communication; weak efficacy of government communication; and strong efficacy of government communication.

\section{Scenario I. Inefficacy of government communication}

When $\beta_{2}=0$, the official message has no positive impact on the rumor spreading among the nonsupporter agents, and this is a scenario of inefficacy for the government communication.

According to the results of Theorems 1 and 2 and Remark 1, the phase portrait of system (5) under scenario I is as shown in Figure 3(a). There are two asymptotically stable nodes $A(1,0)$ and $B(0,1)$, an unstable node $O(0,0)$, and a saddle point $C(m, 1-m)$. These separate $O C$ divide this phase portrait into two regions, region $A O C$, which contains the initial points for which orbits tend to $A(1,0)$, and the region $B O C$, which contains the initial points for which orbits tend to $B(0,1)$. Although coexistence would be the result of initial member sizes on the separate $O C$, this possibility is too sensitive to disturbances to be sociological meaningful. In this scenario, there exists a competitive mechanism between the supporter and nonsupporter agents, and one of them ultimately will win the competition with time evolution. The winner of this competition is determined by the initial density ratio between supporter and non-supporter. If initial density ratio is in the region $A O C$, the non-supporter will disappear, which means that the false rumors end. So, region $A O C$ is called the safety region for information dissemination (SRID). However, if the initial density ratio is in the region $B O C$, then the supporters finally will disappear, which means that the true information will be eliminated in the population, and the society will face a crisis. So, the region $B O C$ is called the unsafety region of information dissemination (URID). On the other hand, Figure 3(a) illustrates that the parameter $m$ value, as a threshold of the rumor spreading competition, decides on the area of the SRID and URID regions, which represents the effect of rumor spreading on change between supporter and nonsupporter agents. 


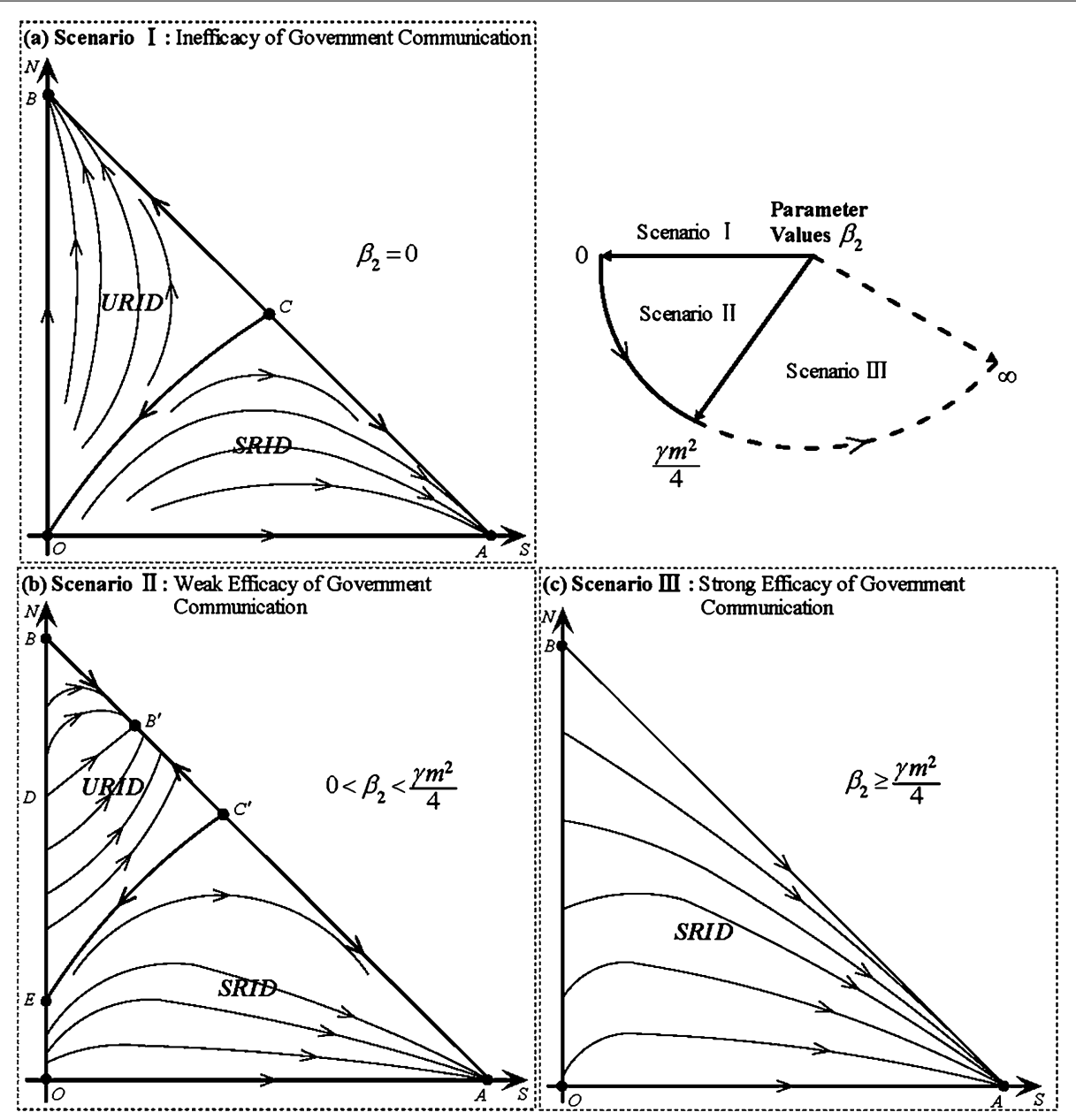

Figure 3 The phase portrait for system (5) under different scenarios.

In general, often at the beginning of such emergency events, nobody, including the government, is able to accurately know the cause, the process, or the losses, so early information is usually muddled or vague, and it is difficult to completely know the truth. Therefore, the false information is often easier to disseminate than the truth, and the initial density of the nonsupporter agent is often greater than that of the supporter agent. That is, the initial density ratio is easily in the URID region, which means that a social crisis may occur under the scenario of inefficacy for the government communication. For example, the spread of rumors in China's SARS Crisis brought seriously negative effects to society.

\section{Scenario II. Weak efficacy of government communication}

When $0<\beta_{2}<\frac{\gamma m^{2}}{4}$, the official message has less influence on public opinion, government communication is weakly effective, and this scenario is defined as a weak efficacy of government communication.

According to the results of Theorems 1, 2, and 3, Figure 3(b) is the phase portrait of system (5) under the scenario II. In this case, there are two asymptotically stable nodes $A(1,0)$ and $B^{\prime}\left(M_{1}, 1-M_{1}\right)$ and a saddle point $C^{\prime}\left(M_{2}, 1-M_{2}\right)$. These separate $C^{\prime} E$ divide this phase portrait into two regions, the region $A O C^{\prime} E$, which contains the initial points for which the orbits tend to $A(1,0)$, and the region $E C^{\prime} B$, which contains the initial points 
for which the orbits tend to $B^{\prime}\left(M_{1}, 1-M_{1}\right)$. Similarly to the preceding scenario, they are called the SRID and URID regions, respectively. If the initial density ratio is in the SRID region, the nonsupporter disappears, and the false rumors end. If the initial density ratio is in the URID region, part of the supporters become nonsupporters, and the proportion of supporters and nonsupporters tends to the constant $\frac{M_{1}}{1-M_{1}}$.

Compared to scenario I, the position of the boundary $C^{\prime} E$ moves up, and the position of the stable nodes $B^{\prime}$ along the boundary $B A$ moves toward the stable nodes $A$ in the phase portrait of system (5), and the SRID area increases and that of the URID decreases because of the increase of the parameter $\beta_{2}$. Therefore, it may be interpreted that the official voice, to some degree, positively affects the rumor spreading and controls public opinion, but ineffective official news dissemination cannot completely eliminate the influence of the false rumor spreading.

\section{Scenario III. Strong efficacy of government communication}

When $\beta_{2} \geq \frac{\gamma m^{2}}{4}$, the official voice has greater influence on the public opinion, and the government communication has strong efficacy. This is a scenario of strong efficacy for the government communication.

According to Theorems 1 and 2, Figure 3(c) is the phase portrait for system (5) with strong government communication efficacy. Unlike the previous scenario, the fixed point $A(1,0)$ is the only asymptotically stable node in the phase portrait. All orbits tend to $A(1,0)$ as $t \rightarrow \infty$, which corresponds to the elimination of the nonsupporter agent and the survival of the supporter agent for all initial density ratios. This means that the official voice has more influence on the public opinion, and rumor spreading is controlled, so all people transform into supporters.

In summary, it may be interpreted that the effective official message dissemination can eliminate false rumors and verify true rumors to break the competitive rumor spreading impact. For example, the Xinhua News Agency, the state press agency of China, released the news to dispel rumors following a falsified report about nuclear leaks in 2012 [25].

\section{Discussion}

In our opinion, a coupling mechanism exists between government communications and the rumor spread after an incident occurs. The official message dissemination influences the rumor-spreading process and affects the public opinion, so it could be seen as an effective measure to positively influence behavior in an emergency to dispel suspicion and reduce irrational behavior [26]. According to our mathematical analysis, the parameters $\beta_{2}, m$, and $\gamma$ influence the evolution of the coupling system (5). The parameter $\beta_{2}$ represents a coefficient for authoritative information to effect on the nonsupporter agents, and parameters $m$ and $\gamma$ donate coefficients for rumor-spreading competition. This result suggests that the official new can, to some extent, impact on true and false rumor-spreading competition, the truth is strengthened, and the falseness gradually dissipates, which in turn breaks the competitiveness between rumor and official information.

The results of the phase portrait of system (5) in Figure 3 suggest that there exist three scenario for agents evolution in terms of the parameter $\beta_{2}$ setting, and they have been defined as government communication inefficacy, weak efficacy, and strong efficacy. In other words, the results show that the degree of government communications can influence the rumor spreading. Effective official messages can reduce the effect of rumors and guide the public opinion. Therefore, in reality, the dissemination of the authority's information needs to be improved to increase its strength for guaranteeing the influence on the 
population after public emergencies. For example, the government could use multiple information distribution platforms, such as the state television, radio, websites, newspapers, and microblogs, to release news about emergencies widely.

Certainly, in the real world, the coupled process between government communication and rumor spreading is very complicated, and it is hard to exactly describe its evolutionary process from a theoretical ad hoc model. To generate effective coping strategies for the government communication in actual emergencies, we still need more theoretical and empirical studies.

\section{Conclusions}

This study extends classical models of spreading epidemics to describe a coupling effect for government communication and rumor spreading after an emergency, which are both key information sources in emergency situations. A coupled system for government communication and rumor spreading was proposed. Mathematical analyses were applied to the proposed model, and the results showed that the official messages have positive influence on true and false rumor-spreading competition. Three different scenarios were identified according to the degree of government involvement to describe the evolution of population behavior under different information streams in an emergency. To conclude, our study may shed new light to comprehend information diffusion in emergencies and provide some hint on how to limit the spreading of rumor within populations when an emergency occurs. Nonetheless, it should be noted that we are dealing with models that are not the reality although to some extent they might help to grasp the reality.

In this model, a closed and homogeneously mixed population was considered. However, in the real world, official message dissemination and the extent of rumor spread may be affected by a more heterogeneous population. Further research could focus on an open heterogeneous population and on the coupling of information dissemination under a complex network environment, such as a scale-free network, which could be useful in specific emergency situations.

Competing interests

The authors declare that they have no competing interests.

Authors' contributions

JX, MZ, and JN designed research; MZ and JN performed research; JX and MZ analyzed data; MZ wrote the paper. All authors read and approved the final manuscript.

Author details

${ }^{1}$ Uncertainty Decision-Making Laboratory, Sichuan University, Yihuan Road, Chengdu, 610064, China. ${ }^{2}$ Department of Mathematics and Physics, Hefei University, Jingxiu Road, Heifei, 230601, China.

Received: 9 December 2015 Accepted: 1 August 2016 Published online: 17 August 2016

References

1. Anthony, S: Anxiety and rumor. J. Soc. Psychol. 89, 91-98 (1973)

2. Nekovee, M, Moreno, Y, Bianconi, G, Marsili, M: Theory of rumour spreading in complex social networks. Physica A $374,457-470$ (2007)

3. Gao, L, Song, C, Gao, Z, Barabási, AL, Bagrow, JP, Wang, D: Quantifying information flow during emergencies. Sci. Rep. 4, 3997 (2014)

4. Galam, S: Modelling rumors: the no plane Pentagon French hoax case. Physica A 320, 571-580 (2003)

5. Zhang, ZL, Zhang, ZQ: An interplay model for rumour spreading and emergency development. Physica A 388 , 4159-4166 (2009)

6. Alexander, DE: Principles of Emergency Planning and Management. Oxford University Press, New York (2002)

7. Regester, M, Larkin, J: Risk Issues and Crisis Management in Public Relations: A Casebook of Best Practice. Kogan Page Publishers, New York (2008)

8. Daley, DJ, Kendall, DG: Epidemics and rumours. Nature 204, 1118 (1964) 
9. Daley, DJ, Gani, J: Epidemic Modelling: An Introduction. Cambridge University Press, Cambridge (2001)

10. Nizamani, S, Memon, N, Galam, S: From public outrage to the burst of public violence: an epidemic-like model. Physica A 416, 620-630 (2014)

11. Lin, T, Fan, C, Liu, C, Zhao, J: Optimal control of a rumor propagation model with latent period in emergency event. Adv. Differ. Equ. 2015, 54 (2015)

12. Zhou, X, Li, X, Wang, WS: Bifurcations for a deterministic sir epidemic model in discrete time. Adv. Differ. Equ. 2014, 168 (2014)

13. Maki, DP, Thompson, M: Mathematical Models and Applications, with Emphasis on Social, Life, and Management Sciences. Prentice Hall, Englewood Cliffs (1973)

14. Xu, JP, Zhang, Y: Event ambiguity fuels the effective spread of rumors. Int. J. Mod. Phys. C 26, 1550033 (2015)

15. Zanette, DH: Dynamics of rumor propagation on small-world networks. Phys. Rev. E 65, 041908 (2002)

16. Moreno, Y, Nekovee, M, Pacheco, AF: Dynamics of rumor spreading in complex networks. Phys. Rev. E 69, 066130 (2004)

17. Huo, LA, Huang, P, Fang, X: An interplay model for authorities' actions and rumor spreading in emergency event. Physica A 390, 3267-3274 (2011)

18. Zhao, L, Wang, Q, Cheng, J, Zhang, D, Ma, T, Chen, Y, Wang, J: The impact of authorities' media and rumor dissemination on the evolution of emergency. Physica A 391, 3978-3987 (2012)

19. May, RM, Anderson, RM: Population biology of infectious diseases: Part II. Nature 280, $455-461$ (1970)

20. Goffman, W: An epidemic process in an open population. Nature 250, 831-832 (1965)

21. Xia, ZY, Huang, LL: Emergence of social rumor: modeling, analysis, and simulations. In: Shi, Y (ed.) Proceedings of the 7th International Conference, Beijing, 27-30 May 2007, pp. 90-97. Springer, Berlin (2007)

22. Hethcote, HW: The mathematics of infectious diseases. SIAM Rev. 42, 599-653 (2000)

23. Han, L, Ma, Z, Hethcote, HW: Four predator prey models with infectious diseases. Math. Comput. Model. 34, 849-858 (2001)

24. Perko, L: Differential Equations and Dynamical Systems. Springer, New York (2013)

25. Commentary: Dispel Rumors After Falsified Nuclear Leak Report. http://news.xinhuanet.com/english/indepth/2012-02/02/c131388825.htm

26. Green, LV, Kolesar, PJ: Improving emergency responsiveness with management science. Manag. Sci. 50, 1001-1014 (2004)

\section{Submit your manuscript to a SpringerOpen ${ }^{\ominus}$ journal and benefit from:}

- Convenient online submission

Rigorous peer review

- Immediate publication on acceptance

- Open access: articles freely available online

- High visibility within the field

- Retaining the copyright to your article 